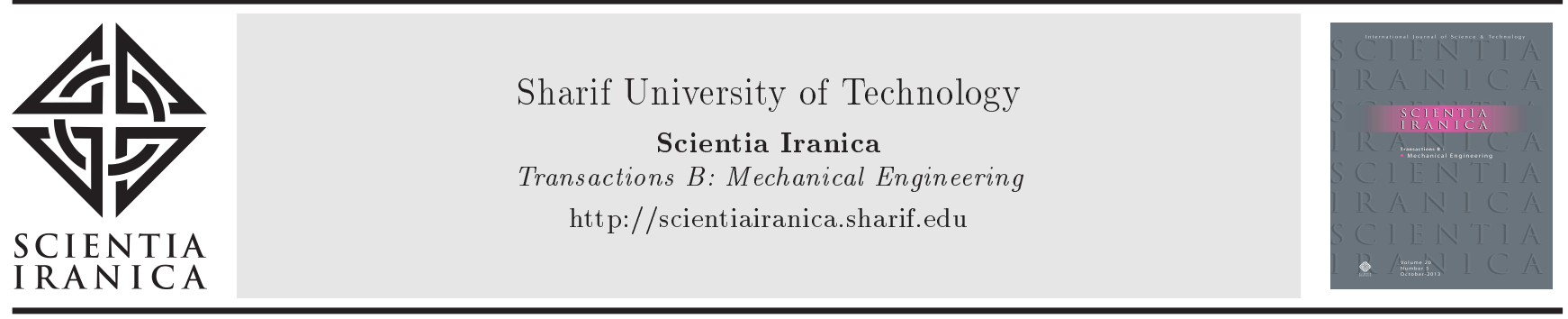

\title{
Plasma based surface modification of poly (dimethylsiloxane) electrospun membrane proper for organ-on-a-chip applications
}

\author{
A. Kiyoumarsioskouei ${ }^{a, b}$, M.S. Saidi ${ }^{a},{ }^{*}, H$. Moghadas $^{a}$, and B. Firoozabadi ${ }^{a}$ \\ a. Department of Mechanical Engineering, Sharif University of Technology, 11365/8639, Tehran, Iran. \\ b. Dalio Institute of Cardiovascular Imaging, Department of Radiology, Weill Cornell Medicine.
}

Received 1 January 2017; received in revised form 3 September 2017; accepted 13 January 2018

\section{KEYWORDS}

Electrospun porous membrane;

Superhydrophilic

surfaces;

Superhydrophobic surfaces;

Cell culture;

Organ on a chip;

Flexible membrane;

Strong membrane;

Surface modifications.

\begin{abstract}
Constructing of the scaffolds for cell culture applications has long been of interest for engineering researchers and biologists. In this study, a novel process is utilized for construction of suitable membrane with a high mechanical strength and appropriate surface behavior. Poly (dimethylsiloxane) (PDMS) is electrospun in fine fibers using poly (methyl methacrylate) (PMMA) as the carrier polymer in different weight ratios. Since the surface behavior of all PDMS substrates is moderately hydrophobic $(120<$ Contact Angle $(C A)<150)$, the electrospun membranes with higher PDMS ratios show slightly higher hydrophilicity. Direct plasma treatment is utilized to change the interfacial wettability of the membrane. Applying plasma changes the surface energy and renders the PDMS/PMMA substrates superhydrophilic $(C A<5)$. In the following, the mechanical properties of the membrane are evaluated by the tensile test, and the results confirm the suitable mechanical strength of electrospun PDMS for cell culturing objectives (yield stress of more than $10 \mathrm{kPa}$ ). Also, changing the PDMS ratio does not significantly affect the total stiffness of the membrane. Thus, applying the proposed fabrication protocol provides a proper platform for cell viability and proliferation, which is proven by the results of MTT assay.
\end{abstract}

(C) 2019 Sharif University of Technology. All rights reserved.

\section{Introduction}

Porous membranes are one of the inseparable parts of most of the "organ-on-a-chip" studies [1-3]. Also, in recent years, the popularity of the electrospun scaffolds in biomedical applications has been increased [4-7] since it is a cost-effective and very flexible method for fabricating two and three-dimensional cell culture scaffolds [8-14]. Due to the fibrous morphology of electrospun scaffolds, surface properties are signifi-

*. Corresponding author. Tel.: +9866165558

E-mail address: mssaidi@sharif.edu (M.S. Saidi). cantly distinct compared with base polymer sheets. Indeed, surface roughness causes the Contact Angle (CA) to increase in electrospun hydrophobic polymers according to the Wenzel's theory. This phenomenon is not favorable in cell culture applications in most of the experiments, because cells need to adhere to the substrate. To address this problem, chemical or electrochemical methods have been used to render such scaffolds hydrophilic [15-17]. Lee et al. [15] used AA-grafted nanofibers to treat the electrospun polyurethane nanofiber matrices surface. The results confirmed that the contact angle of the AA-grafted treated surface was 10 times smaller than the untreated ones. Collagen is another well-known natural polymer, which is commonly found in the extracellular matrix 
as it provides good adhesive properties, and has been frequently used in cell culture applications. Polini et al. [18] functionalized the polymethylmethacrylate (PMMA) electrospun fibers scaffold with collagen by different methods of polymer coating. They demonstrated that the type of linking of collagen to the surface played a more important role than the amount of coating of the collagen in cell adhesion property. More novel works in fabrication of the electrospun biomembrane using other polymers than PDMS have been done as well $[19,20]$. The mechanical properties and fulfilling the tissue engineering requirements are the objectives studied in the mentioned works.

Furthermore, other similar protocols as well as irradiation methods, known as photochemical modifications, have been used [21-23]. Zhang et al. [24] used UV radiation to change the hydrophobicity of the Poly (vinylidene fluoride) (PVF) membrane. They showed that contact angle could be decreased considerably by increasing the radiation time.

From the structural point of view, the mechanical strength plays an important role in the cell culturing membrane selection [25-27]. In most of the applications, the membrane is better to be as thin as possible while it also needs to have good resistance against the mechanical stimulations [11]. Chen et al. [28] measured the Young modulus and ultimate tensile strength of the nanofibers and studied the effect of crosslinking on the mechanical properties of the fibrous membranes. The membrane tensile test was also done by Niu et al. [29] for single PDMS fibers and a fibrous mat made of Poly Vinyl Pyrrolidone (PVP). They showed that elongation of the PDMS fiber mat was more than that of a single PDMS fiber; also, elongation of the single PDMS fiber was more than that of PDMS cast film. Previously, it was indirectly reported that a minimum of about $0.1 \mathrm{~N}$ for mechanical tensile strength was suitable for porous membranes in organ-on-a-chip applications while the membranes thickness was more than $10 \mu \mathrm{m}[30,31]$.

To the best knowledge of the authors, the enhancement of surface wettability of electrospun PDMS base membranes to make them superhydrophilic, which is appealing in cell culture applications, as well as the quantification of mechanical properties of such scaffolds has not been investigated in the literature, which is the primary objective of the present paper. Already, similar studies have been done on PCL or some other polymers [32]. In the present study, the goal is improving the wettability of a prefabricated superhydrophilic PDMS membrane in cell culture applications. First, superhydrophobic porous films with different ratios of PDMS/PMMA are fabricated using electrospinning process. Then, a simple, yet robust, technique will be introduced to render the membranes superhydrophilic. Finally, surface wettability as well as mechanical properties of the fabricated electrospun membranes will be investigated in details.

\section{Materials and methods}

The kit of SYLGARD ${ }^{\circledR} 184$ silicone elastomer supplied from Dow Corning Corporation was used as the base polymer. Also, PMMA (MW 350000) supplied from Sigma-Aldrich Corporation was utilized as a carrier polymer. Tetrahydrofuran (THF) was also supplied from Sigma-Aldrich and used as the solver of the carrier solution. PDMS base polymer was mixed with its curing agent with a ratio of 1:10 (Wt/Wt). PMMA/THF solution was prepared with the ratio of $1: 6(\mathrm{Wt} / \mathrm{Wt})$. Also, for cellular tests, human pulmonary alveolar epithelial cells were cultured in DMEM/F12 media, supplemented with $10 \%$ fetal bovine serum and $2 \%$ penicillin/streptomycin. The Human Epithelial Lung Cells (HELC) were cultured in two different tests. Also, a material of a mixture of $5 \mathrm{mg} / \mathrm{ml}$ MTT solution in the RPMI environment (in 5 vials) was utilized for MTT assay.

\subsection{Electrospinning procedure}

The mixture of the PDMS and PMMA solutions was prepared in different weight ratios $\{0: 1 \quad 1: 1 \quad 2: 1 \quad 3: 1$ 4:1 5:1 6:1 all in Wt(PDMS)/Wt(PMMA) $\}$ and the membranes were fabricated by electrospinning method. The electrospinning process was conducted at the voltage of $12 \mathrm{kV}$ while the nozzle distance was adjusted at $15 \mathrm{~cm}$. The polymer flow rate was set at about $5 \mathrm{~mL} / \mathrm{min}$.

\subsection{Surface characterization}

The quality and topographical features of all membranes were quantified by Scanning Electron Microscopy (SEM). JXA-840 electron microscopy model of the Jeol Japan Company was used to this end.

\subsection{Mechanical strength characterization}

A load cell with the precession of $0.001 \mathrm{~N}$ in a tensile testing machine of the hounsfield h10 ks model of test Resources Company was used. Membrane samples were cut into rectangular samples with the size of about $20(\mathrm{~mm}) \times 5(\mathrm{~mm})$. Hollow quadrangular paper frames were built for better alignment of the samples to the setup [33], as shown in Figure 1.

\subsection{Contact angle measurement}

To measure the contact angle, a manual setup compatible for putting the membrane was prepared.

A high-resolution horizontal camera was installed in front of the stage, and a light source was mounted on the backside. Figure 2 shows a schematic view of the contact angle measurement device. 


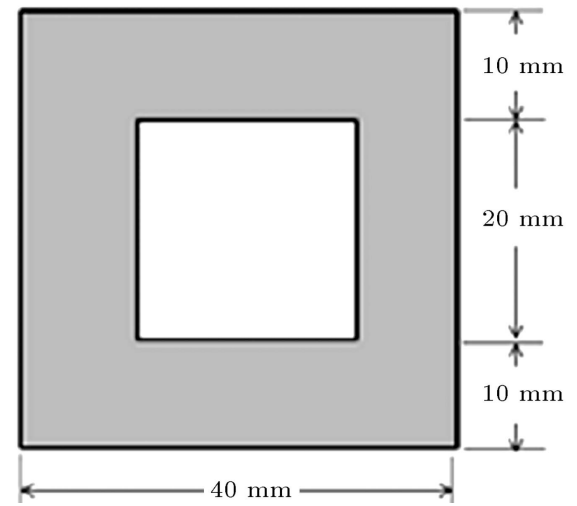

Figure 1. Quadrangular hollow paper for tensile test frame. This framework was utilized for mounting of the samples on the machine.

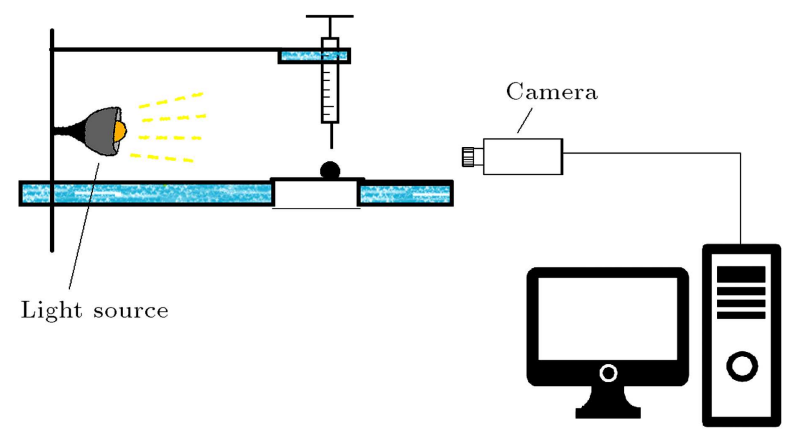

Figure 2. Setup for measuring the contact angle. The syringe was schematic, and it could be replaced by pipette.

\section{Results and discussion}

The generated electrospun membranes exhibited good appearance and were uniform. The Scanning Electron Microscopy (SEM) image of an electrospun membrane is shown in Figure 3.

\subsection{Surface modification}

The applications of PDMS-based materials are significantly increasing in biomedical sciences. Intrinsically, the polymer sheet is slightly hydrophobic $(90<C A<$ 120) [34]. Using oxygen plasma has beneficial effects on decreasing the contact angle and, subsequently, increasing the hydrophilicity. Widespread experiments

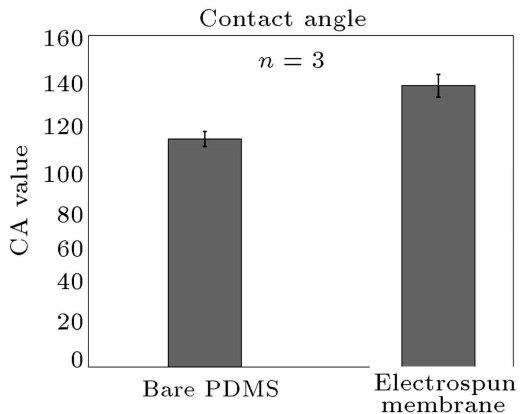

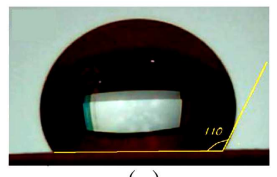

(a)

(b)

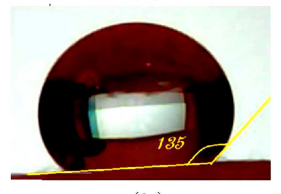

Figure 4. High-resolution image of distilled water droplet on (a) sheet of the PDMS, and (b) electrospun PDMS membrane.

have shown decrease in the contact angle of about $30^{\circ}$ [35-37], which is helpful in microfluidic applications, but no work is found to report a reduction in contact angle to a highly hydrophilic phase.

The electrospinning of the polymer seems to increase hydrophobicity. Electrospinning changes porosity and roughness of the membrane, which is a valid parameter in wettability [38]. Figure 4 displays the contact angles in two different conditions. This phenomenon could be explained using Wenzel's theory. According to the Wenzel's model, when the droplet size is sufficiently larger than the surface roughness, increasing the roughness ratio causes increase in contact angle of the surface [39]. Although the porous property and the surface roughness of the electrospinning scaffolds are considered as suitable parameters, increasing the contact angle is a negative factor in cell adhesion event. To modify the surface, plasma treating procedure was used. Two protocols were utilized. In the first procedure, the classical method of $1 \mathrm{~min}$ plasma exposure by plasma generator was used. In the second procedure, the exposure time was decreased to 7 seconds, but the number of exposures was tripled (this protocol was obtained from about 200 trial and error tests). Figure 5 displays the effect of plasma treatment on the electrospun PDMS membrane. Decreasing the exposure time does not provide enough time to modify the surface of the membrane. On the other hand, increasing the exposure time causes a different chemical

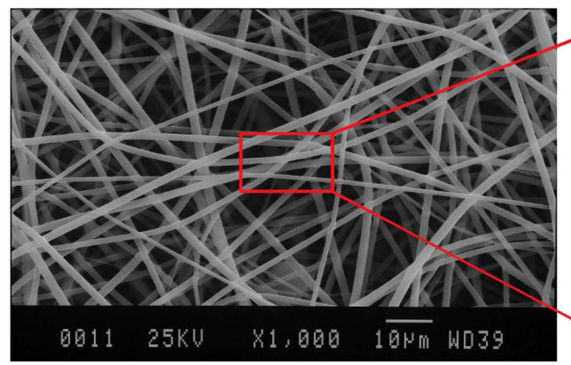

(a)

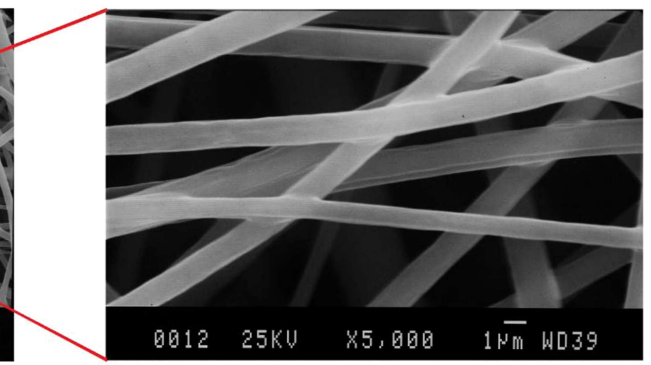

(b)

Figure 3. Scanning electron microscopy image of the electrospun PDMS membrane: (a) $1000 \times$ and (b) $10000 \times$. 


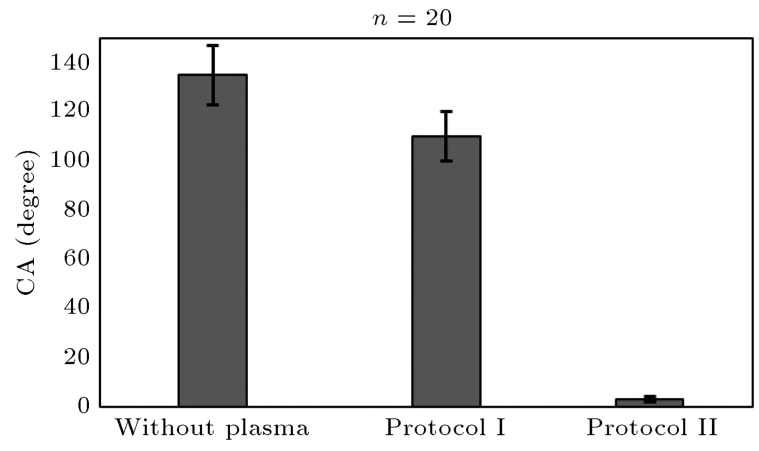

Figure 5. Measured contact angle in different conditions. Comparison of the contact angle before surface modification was done with different modified surfaces.

reaction on the surface of fibers [40] and increase in carbonaceous in the surface, which is not a positive event in rendering the surface hydrophilic. Due to this, increasing the exposure number was proposed and used in the new protocol.

According to the experiments, it is concluded that change in the roughness of the surface, which happens due to electrospinning process, causes the slightly hydrophobic $(90<C A<120)$ membranes to change into moderately hydrophobic $(120<C A<$ $150)$ and even sometimes to superhydrophobic $(150<$ $C A<180$ ) phases (as mechanical changes take place on the membrane surface). This phenomenon is caused by changing surface roughness in micro scale when the fibers are accumulated in spinning. As described before, changing the surface roughness causes the hydrophobicity of the membrane to increase (Wenzel's model). The offered protocol modifies the hydrophobic surfaces to hydrophilic phases and, consequently, the superhydrophobic surfaces to superhydrophilic ones.

\subsection{Tensile test}

Tensile tests were utilized to evaluate the effect of PDMS concentrations on the mechanical behavior of the electrospun membrane. The ratios of the solutions are shown in Table 1 . The yield strength and elongation of the membrane were measured for all seven samples, which are shown in Figures 6 and 7

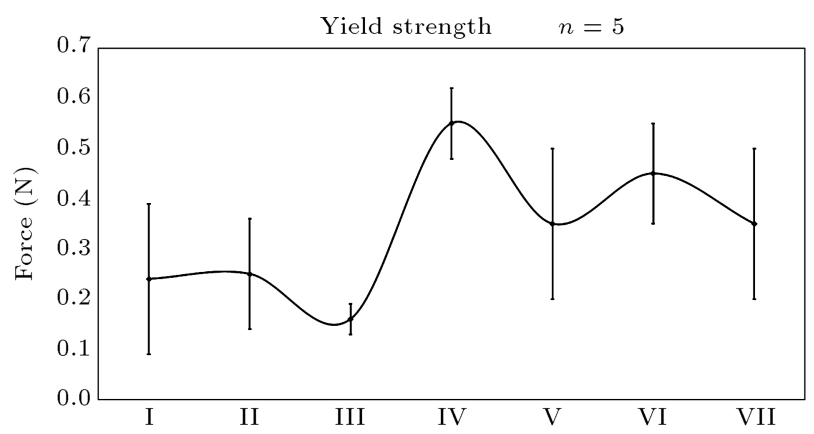

Figure 6. The yield strength of the electrospun membrane with different PDMS percentages.

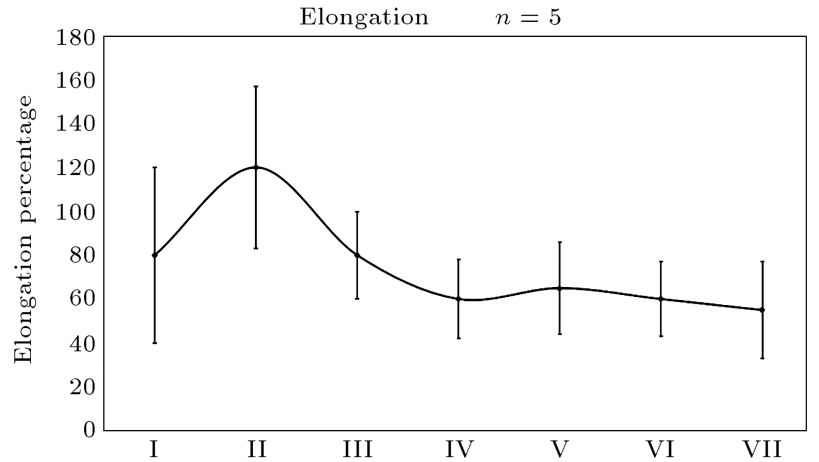

Figure 7. Elongation of the electrospun membrane with different PDMS percentages.

Table 1. Sample naming according to the ratio of the PDMS/PMMA.

\begin{tabular}{cc}
\hline Sample number & Ratio of PDMS / P1VEMA \\
\hline I & $0: 1$ \\
II & $1: 1$ \\
III & $2: 1$ \\
IV & $3: 1$ \\
V & $4: 1$ \\
VI & $5: 1$ \\
VII & $6: 1$ \\
\hline
\end{tabular}

for different PDMS percentages. Considering these two figures, it could be concluded that the stiffness and elongation of membrane are appropriate for cell culture applications and, consequently, for organ-on-achip applications according to references [41-44].

Table 1 shows the samples which were used for the tensile test. Ratio of the PDMS/PMMA is shown. All the samples have the thickness of $50 \pm 10 \mu \mathrm{m}$.

Also, it is observed that the mechanical behavior of the membrane is not a linear function of the ratio of the PDMS. Although the spinning conditions and the thickness where the fibers are bounded by each other affect the mechanical stiffness of the membrane and different mechanical behaviors are observed, all the samples are appropriate for organ-on-a-chip applications $[30,31,42]$.

\subsection{Cytocompatiblity}

The capability of the membrane for culturing the cells is compared with the classical cell cultures treated wells by utilizing MTT assay. Since the MTT reagent was sensitive to light, the whole environment was isolated from the light source. To do the assay, first, the cells medium was discharged from vials, then $400 \mu \mathrm{l}$ of PRMI and $10 \mu \mathrm{l}$ of MTT solutions were added to the whole 24 well plates. The plates were placed in the incubator for 24 hours. In the next step, the 


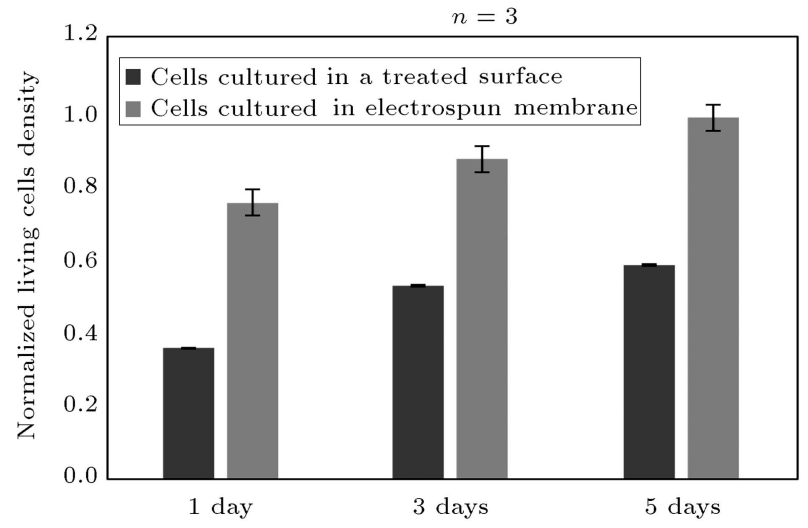

Figure 8. The cells aggregation evaluation using MTT assay (normalized to the maximum value of measurement).

MTT solution was aspirated off and $1 \mathrm{ml}$ of acidified isopropanol solution was replaced in each well (see Figure 8).

Pipetting was done slowly and the well was put in the incubator for about 30 minutes. After doing this procedure, a $510 \mathrm{~nm}$ wavelength spectrophotometer was used to measure the light intensity. The assays were done three times and the results were averaged. The electrospun membrane provided a better condition for cell culturing. The intensity processing represented the viability of the cells in the test. The results are displayed in Figure 8. They show that the cells have better viability and proliferation performance.

\section{Conclusions}

The electrospinning based fabrication of a flexible beadless superhydrophobic membrane was reported in this study. Electrospinning caused a remarkable increase in hydrophobicity of the surface. This could be attributed to the increase in the roughness and creation of nano-metric porosities on the surface of the polymer. The results showed that physical increase in the hydrophobicity of the membrane enhanced the potential of the surface going to a more hydrophilic phase using oxygen plasma treatment. Also, the mechanical strength of the membrane was studied as another important characteristic of the scaffolds. Although the strength of the membrane was not a linear function of the PDMS ratios, it was sufficient for organ-on-a-chip applications.

The capability of the membrane for cell adhesion was tested. The cells adhered to the membrane without using any extracellular matrix polymers. Also, cells viability was tested using MTT assay. Results showed that the cells cultured on the electrospun membrane had about two times more aggregation capabilities. The cells could be alive and proliferate better than the ones on the treated vials. Finally, this work showed that manufacturing electrospun PDMS/PMMA mem- brane was a fast, biocompatible, flexible, and costeffective method for cell culture and organ-on-a-chip applications.

\section{References}

1. Bhatia, S.N. and Ingber, D.E. "Microfluidic organs-onchips", Nature, 201, p. 4 (2014).

2. Huh, D., Kim, H.J., Fraser, J.P., et al. "Microfabrication of human organs-on-chips", Nature Protocols, 8(11), pp. 2135-2157 (2013).

3. Zheng, F., Fu, F., Cheng, Y., Wang, C., Zhao, Y., and Gu, Z. "Organ-on-a-Chip Systems: Microengineering to Biomimic Living Systems", Small (2016).

4. Volova, T., Goncharov, D., Sukovatyi, A., Shabanov, A., Nikolaeva, E. and Shishatskaya, E., "Electrospinning of polyhydroxyalkanoate fibrous scaffolds: effects on electrospinning parameters on structure and properties", Journal of Biomaterials Science, Polymer Edition, 25, pp. 370-393 (2014).

5. Shankhwar, N., Kumar, M., Mandal, B.B., Robi, P., and Srinivasan, A. "Electrospun polyvinyl alcoholpolyvinyl pyrrolidone nanofibrous membranes for interactive wound dressing application", Journal of Biomaterials Science, Polymer Edition, 27, pp. 247-262 (2016).

6. Yao, C., Li, X., and Song, T. "Fabrication of zein/hyaluronic acid fibrous membranes by electrospinning", Journal of Biomaterials Science, Polymer Edition, 18, pp. 731-742 (2007).

7. Khajavi, R. and Abbasipour, M. "Electrospinning as a versatile method for fabricating coreshell, hollow and porous nanofibers", Scientia Iranica, 19, pp. 2029-2034 (2012).

8. Carletti, E., Motta, A., and Migliaresi, C. "Scaffolds for tissue engineering and 3D cell culture", 3D Cell Culture: Methods and Protocols, 695, pp. 17-39 (2011).

9. Heydarkhan-Hagvall, S., Schenke-Layland, K., Dhanasopon, A.P., et al. "Three-dimensional electrospun ECM-based hybrid scaffolds for cardiovascular tissue engineering", Biomaterials, 29, pp. 2907-2914 (2008).

10. Li, M., Mondrinos, M.J., Gandhi, M.R., Ko, F.K., Weiss, A.S., and Lelkes, P.I. "Electrospun protein fibers as matrices for tissue engineering", Biomaterials, 26, pp. 5999-6008 (2005).

11. Pham, Q.P., Sharma, U., and Mikos, A.G. "Electrospinning of polymeric nanofibers for tissue engineering applications: a review", Tissue Engineering, 12, pp. 1197-1211 (2006).

12. Sharma, Y., Tiwari, A., Hattori, S., et al. "Fabrication of conducting electrospun nanofibers scaffold for threedimensional cells culture", International Journal of Biological Macromolecules, 51, pp. 627-631 (2012). 
13. Wallin, P., Zandén, C., Carlberg, B., Erkenstam, N. H., Liu, J., and Gold, J. "A method to integrate patterned electrospun fibers with microfluidic systems to generate complex microenvironments for cell culture applications", Biomicrofluidics, 6, p. 024131 (2012).

14. Zonooz, N.F. and Salouti, M. "Extracellular biosynthesis of silver nanoparticles using cell filtrate of streptomyces sp. ERI-3", Scientia Iranica, 18, pp. 1631-1635 (2011).

15. Lee, K.H., Kwon, G.H., Shin, S.J., et al. "Hydrophilic electrospun polyurethane nanofiber matrices for hMSC culture in a microfluidic cell chip", Journal of Biomedical Materials Research, Part A, 90, pp. 619-628 (2009).

16. Rupp, F., Scheideler, L., Olshanska, N., De Wild, M., Wieland, M., and Geis-Gerstorfer, J. "Enhancing surface free energy and hydrophilicity through chemical modification of microstructured titanium implant surfaces", Journal of Biomedical Materials Research, Part A, 76, pp. 323-334 (2006).

17. Théry, M., Racine, V., Pépin, A., et al. "The extracellular matrix guides the orientation of the cell division axis", Nature Cell Biology, 7, pp. 947-953 (2005).

18. Polini, A., Pagliara, S., Stabile, R., et al. "Collagenfunctionalised electrospun polymer fibers for bioengineering applications", Soft Matter, 6, pp. 1668-1674 (2010).

19. Pauly, H.M., Kelly, D.J., Popat, K.C., et al. "Mechanical properties and cellular response of novel electrospun nanofibers for ligament tissue engineering: Effects of orientation and geometry", Journal of the Mechanical Behavior of Biomedical Materials, 61, pp. 258-270 (2016).

20. Walser, J. and Ferguson, S.J., "Oriented nanofibrous membranes for tissue engineering applications: Electrospinning with secondary field control", Journal of the Mechanical Behavior of Biomedical Materials, 58, pp. 188-198 (2016).

21. Brink, L., Elbers, S., Robbertsen, T., and Both, P. "The anti-fouling action of polymers preadsorbed on ultrafiltration and microfiltration membranes", Journal of Membrane Science, 76, pp. 281-291 (1993).

22. Ulbricht, M., Matuschewski, H., Oechel, A., and Hicke, H.-G. "Photo-induced graft polymerization surface modifications for the preparation of hydrophilic and low-proten-adsorbing ultrafiltration membranes", Journal of Membrane Science, 115, pp. 31-47 (1996).

23. Ulbricht, M., Oechel, A., Lehmann, C., Tomaschewski, G., and Hicke, H.G. "Gas-phase photoinduced graft polymerization of acrylic acid onto polyacrylonitrile ultrafiltration membranes", Journal of Applied Polymer Science, 55, pp. 1707-1723 (1995).

24. Zhang, M., Nguyen, Q.T., and Ping, Z. "Hydrophilic modification of poly (vinylidene fluoride) microporous membrane", Journal of Membrane Science, 327, pp. 78-86 (2009).
25. Yi, F., Lu, J.-W., Guo, Z.-X., and Yu, J. "Mechanical properties and biocompatibility of soluble eggshell membrane protein/poly (vinyl alcohol) blend films", Journal of Biomaterials Science, Polymer Edition, 17, pp. 1015-1024 (2006).

26. Su, Y., Chen, C., Li, Y., and Li, J. "Preparation of PVDF membranes via TIPS method: the effect of mixed diluents on membrane structure and mechanical property", Journal of Macromolecular Science, Part A: Pure and Applied Chemistry, 44, pp. 305-313 (2007).

27. Mazza, E., Ganghoffer, J.-F., and Ehret, A.E. "Mechanics of biological membranes", Journal of the Mechanical Behavior of Biomedical Materials, 58, p. 1,5 (2016).

28. Chen, J.-P., Chang, G.-Y., and Chen, J.-K. "Electrospun collagen/chitosan nanofibrous membrane as wound dressing", Colloids and Surfaces A: Physicochemical and Engineering Aspects, 313, pp. 183-188 (2008).

29. Niu, H., Wang, H., Zhou, H., and Lin, T. "Ultrafine PDMS fibers: preparation from in situ curingelectrospinning and mechanical characterization", Rsc Advances, 4, pp. 11782-11787 (2014).

30. Huh, D., Matthews, B.D., Mammoto, A., MontoyaZavala, M., Hsin, H.Y., and Ingber, D.E. "Reconstituting organ-level lung functions on a chip", Science, 328, pp. 1662-1668 (2010).

31. Jang, K.-J. and Suh, K.-Y. "A multi-layer microfluidic device for efficient culture and analysis of renal tubular cells", Lab on a chip, 10, pp. 36-42 (2010).

32. Li, Y., Thouas, G.A., and Chen, Q. "Novel elastomeric fibrous networks produced from poly (xylitol sebacate) 2: 5 by core/shell electrospinning: fabrication and mechanical properties", Journal of the Mechanical Behavior of Biomedical Materials, 40, pp. 210-221 (2014).

33. Tech, E. "Tensile testing of electrospun nanofiber membrane", ES2002 version 1 Ed. (2013).

34. Qiu, W., Sun, X., Wu, C., Hjort, K., and Wu, Z. "A contact angle study of the interaction between embedded amphiphilic molecules and the PDMS matrix in an aqueous environment", Micromachines, 5, pp. 515-527 (2014).

35. Morra, M., Occhiello, E., Marola, R., Garbassi, F., Humphrey, P., and Johnson, D. "On the aging of oxygen plasma-treated polydimethylsiloxane surfaces", Journal of Colloid and Interface Science, 137, pp. 1124 (1990).

36. Owen, M.J. and Smith, P.J., "Plasma treatment of polydimethylsiloxane", Journal of Adhesion Science and Technology, 8, pp. 1063-1075 (1994).

37. Tezuka, Y., Fukushima, A., Matsui, S., and Imai, K. "Surface studies on poly (vinyl alcohol)-poly (dimethylsiloxane) graft copolymers", Journal of Colloid and Interface Science, 114, pp. 16-25 (1986). 
38. Khorasani, M., Mirzadeh, H., and Kermani, Z. "Wettability of porous polydimethylsiloxane surface: morphology study", Applied Surface Science, 242, pp. 339345 (2005).

39. Patankar, N.A. "Hydrophobicity of surfaces with cavities: making hydrophobic substrates from hydrophilic materials?", Journal of Adhesion Science and Technology, 23, pp. 413-433 (2009).

40. Borcia, C., Borcia, G., and Dumitrascu, N. "Surface treatment of polymers by plasma and UV radiation", Romanian Journal in Physics, 56, pp. 224-232 (2011).

41. Gilbert, J., Weinhold, P.S., Banes, A., Link, G., and Jones, G. "Strain profiles for circular cell culture plates containing flexible surfaces employed to mechanically deform cells in vitro", Journal of Biomechanics, 27, pp. 1169-1177 (1994).

42. Hampton, C., Webster, G.D., Rzigalinski, B., and Gabler, H.C. "Mechanical properties of polytetraflouroethylene elastomer membrane for dynamic cell culture testing", Biomedical Sciences Instrumentation, 44, pp. 105-110 (2007).

43. Madihally, S.V. and Matthew, H.W. "Porous chitosan scaffolds for tissue engineering", Biomaterials, 20, pp. 1133-1142 (1999).

44. Niknejad, H., Peirovi, H., Jorjani, M., Ahmadiani, A., Ghanavi, J., and Seifalian, A.M. "Properties of the amniotic membrane for potential use in tissue engineering", Eur Cells Mater, 15, pp. 88-99 (2008).

\section{Biographies}

Amir Kiyoumarsioskouei received his BS and MS in Mechanical Engineering mainly working on environmental and energy researches. He started his $\mathrm{PhD}$ at Sharif University of Technology in 2012 and extended his researches in Weill Cornell Medical College from June 2016. He did the fabrication and most of experiments in this work.

Mohammad Said Saidi is a Professor in Mechanical Engineering at Sharif University of Technology. He completed his $\mathrm{PhD}$ in Massachusetts Institute of Technology (MIT). He was the main manager and idea developer in the recent work.

Hajar Moghadas received her BS and MS in Mechanical Engineering. She started her $\mathrm{PhD}$ at Sharif University of Technology in 2012 and, due to her expertise, she helped in the cell culture works in the recent work.

Bahar Firoozabadi is a Professor in Mechanical Engineering at Sharif University of Technology. She completed her $\mathrm{PhD}$ at Sharif University. She was a very impressive consultant in the entire period of this project. 